Chapter 2

\title{
Indications for Brain Computed Tomography Scan After Mild Traumatic Brain Injury
}

\author{
Shayan Abdollah Zadegan and \\ Vafa Rahimi-Movaghar \\ Additional information is available at the end of the chapter \\ http://dx.doi.org/10.5772/57308
}

\section{Introduction}

Trauma to the head may cause damage to the brain parenchyma. This intracranial damage is more probable in severe than mild forms of head trauma. Despite this knowledge, physicians could not disregard the risk of brain injury in mild forms; thus, in many cases of head trauma, computed tomography (CT) scans are performed to detect possible intracranial injuries. This excessive CT scanning could cause unnecessary radiation exposure for patients, many cases of which are children and impose a heavy burden on the society. Different studies tried to decrease unnecessary CT scans by establishing clinical rules to predict possible injuries. These rules will help clinicians to safely recognize at risk patients with clinical signs and symptoms and perform CT scans when it is really necessary. The need for these rules is more prominent in mild forms of traumatic brain injury, where patients usually come with normal level of consciousness. This chapter will introduce different clinical studies related to indications for brain CT scan after Mild traumatic brain injury.

\section{Mild Traumatic Brain Injury (MTBI)}

When it comes to mild traumatic brain injury (MTBI), general expectation is a self-limited situation with no intracranial structural damages. Well, it is not true. According to the American Congress of Rehabilitation Medicine (ACRM) and World Health Organization (WHO), MTBI is described as Glasgow Coma Scale (GCS) score of 13 to 15 due to brain damage resulting from blunt trauma or acceleration/deceleration forces. One or more of the following symptoms and signs could be accompanied by MTBI: "confusion/disorientation, loss of 
consciousness for $30 \mathrm{~min}$ or less, posttraumatic amnesia for less than $24 \mathrm{hr}$, and/or other transient neurological abnormalities such as focal signs, seizure, and intracranial lesion" [1]. Some other signs and symptoms like headache, dizziness, irritability, lethargy, vomiting, blurred vision, fatigue, and poor concentration have also been reported [1-5]. However, the definition has been changing for the last decade and there is no fully agreed definition of MTBI [6]. Also, there is a body of evidence suggesting that MTBI could be a misnomer, because in traumatic brain injury (TBI) cases with GCS score of 13-15, some structural changes and longterm sequels are documented. However the rate of neurosurgical intervention is very low and the effect of these structural changes on long-term prognosis is not fully understood [7-11]. So, the term "complicated MTBI" was suggested by some authors to fill this defect, but this area is still challenging [8;12]. Possible pathological features including intracranial hemorrhage, diffuse axonal injury and brain contusion might be present in MTBI and could be detected by different neuroimaging modalities. The first-line modality is computed tomography (CT) scan; skull radiographs are not recommended as a useful screen test in TBI due to low sensitivity and misleading false negatives [13]. Emergency head CT studies showed that up to $15 \%$ of head trauma patients with GCS score of 15 could have acute lesions; however, the rate of neurosurgical intervention in these patients is very low (less than 1\%) [13-22]. In consequence, there is a chance to reduce unnecessary imaging studies in MTBI with certain clinical rules. Using such rules could reduce imaging charges, radiation exposure and time in emergency departments (ED) $[2 ; 6 ; 8 ; 12 ; 13 ; 15 ; 23]$. In this chapter, we will focus on the most frequently used clinical rules for reducing CT scans after MTBI.

\section{Leading clinical rules}

The most validated rule in minor head injury is the Canadian CT head rule (CCHR), presented by Stiell and colleagues in 2001 [24]. Their prospective cohort study was conducted in ED of 10 Canadian large hospitals and 3,121 patients were enrolled. The enrollment criteria were based on MTBI description. They also excluded patients below 16 years old and those with minimal injuries (no loss of consciousness, amnesia, or disorientation), primary events like primary seizure or syncope, obvious penetrating skull injury or depressed fracture, acute focal neurological deficit, unstable vital signs, bleeding disorder or used oral anticoagulants and pregnancy as well as patients returned for reassessment of the same head injury. The main outcome measures for this rule were the need for neurological intervention and clinically important brain injury (detected by CT scan). From 3,121 patients, 2,078 underwent CT scanning according to the clinical judgment of the physician and the remaining 1,043 patients underwent the 14 day telephone proxy outcome measure. High risk patients in this guideline were patients with GCS score of less than 15 (2 hours after injury), suspected open or depressed skull fracture, signs of basal skull fracture (hemotympanum, 'raccoon' eyes, cerebrospinal fluid otorrhea/rhinorrhea, Battle's sign), two or more episodes of vomiting and elderly patients ( $\geq 65$ years). The medium risk group in this criteria include patients with more than 30 minutes of amnesia before impact and dangerous mechanism of injury (like pedestrian struck by motor vehicle, occupant ejected from motor vehicle, fall from height $>3$ feet or five stairs) [24] (see 
table 1). It was suggested that patients with any of the high-risk factors would probably need neurosurgical intervention and the CT is necessary in this group. Those MTBI patients with medium risk factors may have a lesion that could be seen on CT scan but probably do not require neurosurgical intervention (even with either of the two medium risk factors) and managing these patients could be performed by CT scan or direct monitoring according to local policy. The sensitivity of the test was 100\% for high-risk factors and $984 \%$ for mediumrisk factors. The test was $496 \%$ specific [24].

\begin{tabular}{ll}
\hline High-risk criteria* & - GCS score $<15$ \\
& - Suspected open or depressed skull fracture \\
& - Signs of basal skull fracture (hemotympanum, 'raccoon' eyes, cerebrospinal fluid \\
& otorrhea/rhinorrhea, Battle's sign) \\
- & Vomiting $\geq 2$ episodes \\
- & Elder patients ( $\geq 65$ years) \\
\hline - Amnesia before impact ( $>30$ min) \\
- Dangerous mechanism of injury \\
$\qquad$ Pedestrian struck by motor vehicle \\
$\bullet$ Occupant ejected from motor vehicle \\
$\bullet$ Fall (>3 feet or five stairs)
\end{tabular}

* Neurosurgical intervention is likely to be needed in high-risk group and a head CT scan is mandatory.

** Medium-risk group probably do not require neurosurgical intervention and could be managed by CT scanning or direct monitoring.

Table 1. The Canadian CT head rule (CCHR) for brain CT scan. (Originally described by Stiell et al. Reproduced with permission [24])

After the Stiell's study, many researchers started to validate the CCHR rule. The results were very diverse. Few studies came up with great questions in effectiveness of CCHR [25;26]. Even increased numbers of CT scans were reported in one British study. The study was a retrospective case note review. This study design may raise some questions about recording all important clinical variables. However, the authors of the article were confident about data collection due to standardized forms that were used in their hospital [25]. Another retrospective study in Australia showed that CCHR would decrease the number of CT scanning without missing the patients requiring neurosurgical intervention but in this study, it missed two patients with clinically significant CT abnormality. Similar to the previous, this study was retrospective and relies on the accuracy of medical records [26].

Despite these studies, a larger number of studies found it beneficial or cost-effective to replace current protocols with CCHR [27-35]. A prospective cohort study with 4,551 patients from 10 hospital EDs of the United States reported that CCHR could accurately identify all patients requiring neurosurgical intervention [30]. Another multicenter prospective validation study was conducted in 4 university hospitals of the Netherlands. Among 3,181 patients, CCHR identified all cases requiring neurosurgical intervention (sensitivity of $100 \%$ ); however, it did 
notshow highsensitivity forneurocranial lesions of CTscan (approximately 85\%). The specificity of the test was $37.2 \%-39.7 \%$. This study did not use the exact predictors that Stiell defined previously. For instance, they evaluated GCS of patients at 1 hour after presentation instead of 2 hours. They also used post-traumatic amnesia of more than 30 minutes as a risk factor instead of retrograde amnesia and vomiting was defined as any period of emesis instead of more than 1 episode of emesis [17]. Another cost effective analysis research showed the cost effectiveness of CCHR with US $\$ 120$ million savings annually. However, this finding will be valid only if the CCHR is highly sensitive for neurosurgical intervention prediction and at sensitivities lower than $97 \%$, it will be more cost-effective to perform CT scan for all patients [36].

There are also few trials that aimed to implement CCHR at emergency departments [37;38]. In the opinion of Stiell and colleagues [38], the failure of such trials was the result of physicians' non-compliance. Some examples of such non-compliance of the physicians were forgetting the details of the rules, not believing in the nature of the research, being forced to order CT scans due to patient/family expectations, having inconsistencies among different services in ordering CT scans and insufficient time in a busy ED and finally the idea that the application of the rule would either take too much time or would not be safe for the patients [38]. Other epidemiological studies have confirmed this opinion [39;40].

Two other famous criteria are the NEXUS-II (National Emergency X-Ray Utilization Study) rule and the NOC (New Orleans Criteria). According to NOC, presented by Haydel and colleagues in 2000 [15], minor head injury is defined as loss of consciousness in patients with normal cranial nerves, normal strength and sensation in arms and legs and a GCS score of 15 at arrival. Red flags for head injury are the following seven factors: headache, vomiting, age, drug or alcohol intoxication, short-term memory deficit, physical evidence of trauma above the clavicles, and seizure. These seven factors showed a sensitivity of $100 \%$ for detecting patients with positive CT scans, negative predictive value of $100 \%$ and specificity of $25 \%$. Coagulopathy is absent in NOC because in the first phase of the study conducted by Haydel and colleagues, only 1 patient out of 520 had coagulopathy; so, the researchers could not evaluate this item [15]. Also, imaging for all patients with evidence of trauma above the clavicles would be excessive, because small contusions or lacerations and minor facial injuries would be included and this will increase the rate of CT imaging. Prospective evaluation of NOC showed lower reduction rate of CT scanning and lower specificity (3\% to 31\%) compared to CCHR (48\% to 77\% for high-risk criteria and $37 \%$ to $48 \%$ for high and medium-risk criteria), but the sensitivity of NOC was similar to CCHR (99\% to $100 \%$ ) [16;17;26;32;33;41-43]. Although a 2013 research by Bouida and colleagues in Tunisia has reported a slightly lower sensitivity for NOC ( $86 \%$ sensitivity for clinically significant head CT findings and $82 \%$ sensitivity for the need for neurosurgical intervention) [34]. This difference in sensitivity could be the result of NOC application in GCS lower than 15. As mentioned above, NOC was defined for MTBI patients with GCS scores of not lower than 15. This conclusion will be strengthened by the knowledge that the difference was diminished in a subgroup of patients with GCS score of 15 [34].

In 2002, a prospective multicenter study called NEXUS-II (National Emergency X-Radiography Utilization Study II) was designed to prepare a decision rule for CT imaging of patients with acute blunt head trauma [23;44]. NEXUS II decision instrument enrolled 13,728 patients and presented eight factors significantly associated with intracranial injuries: 1 . evidence of 
significant skull fracture, 2. hematoma of scalp, 3. neurological deficit, 4. altered level of alertness, 5. abnormal behavior, 6. coagulopathy, 7. persistent vomiting and 8. patients aged 65 years or more [23]. NEXUS II criteria provided guidance for patients without any episode of loss of consciousness. This great advantage was absent in the two previous criteria. A recent prospective cohort study reported a sensitivity of $95.1 \%$ and specificity of $41.4 \%$ for neurosurgical intervention for NEXUS II criteria. This study showed that NEXUS II criteria were less sensitive but more specific than NOC and CCHR (sensitivity of $100 \%$ for NOC and CCHR and specificity of $38.3 \%$ for CCHR and $20.4 \%$ for NOC were documented). The potential reduction rate in CT scanning was higher in NEXUS II (39.6\%) compared to CCHR (27\%) and NOC (20.2\%) [45]. Another retrospective analysis reported the sensitivity and specificity of NEXUS II criteria at respectively $97 \%$ (versus $99 \%$ sensitivity for CCHR and NOC) and $47 \%$ (versus specificity of $47 \%$ for CCHR and 33\% for NOC) for detecting any lesions. The sensitivity of NEXUS II criteria for detecting hematoma was higher than the two other criteria (100\% vs. $99 \%$ ); however, researchers conducting the study could not demonstrate that this higher sensitivity was statistically significant [43]. In conclusion, NEXUS II criteria showed the highest reduction rate for CT scanning compared to the other two rules, but it failed to recognize some patients requiring neurosurgical intervention. Table 2 shows all characteristics included in the three above-mentioned leading rules.

\section{Other clinical rules}

Other studies conducted on indications of brain CT scan after MTBI are briefly cited. In 1993, Reinus et al. [46] developed criteria by reviewing medical records and CT scans of 373 patients. The four variable criteria were positive neurologic examination, intoxication, amnesia and a history of focal neurologic deficit. The criteria had a sensitivity of $90.1 \%$ and a negative predictive value of $98.1 \%$ for detecting abnormality in CT scan. The criteria missed 4 patients. None of them needed neurosurgical intervention [46]. In 1994, Duus and colleagues [47] performed a prospective cohort study on 2,204 patients in Denmark. They admitted patients with confusion or aggression in ED, impaired consciousness, focal neurological sign, suspected skull fracture in a clinical exam, alcohol intoxication or other medical conditions that interfere withassessment, history of convulsions, amnesia (morethan 15 minutes), history of unconsciousness (more than 15 minutes and witnessed by a competent observer), 3-year-old or younger children with symptoms and no responsible adult at home. The study proposed the use of the guideline instead of skull radio graphs [47].

In 1995, in a retrospective descriptive study of 1,448 American patients with GCS score of 13 or more, Borczuk [48] found the following factors as high-risk: soft tissue injury, focal neurologic deficit, signs of basilar skull fracture and being older than 60 years. The sensitivity was $91.6 \%$ and specificity was $46.2 \%$ for detecting a CT abnormality. None of the patients missed by these criteria, required medical or neurosurgical management [48]. In 1997, Miller et al. [49] enrolled 2143 patients with the GCS score of 15 and a history of loss of consciousness (LOC) to develop simple clinical criteria to safely reduce the number of CT scans. Miller's 4 high-risk criteria included headache, nausea, vomiting and signs of depressed skull fracture. The criteria 
showed $61 \%$ reduction in the number of CT scans and also identified all patients who required neurosurgical intervention. Holmes et al. examined the criteria in patients with a lower GCS score (GCS=14). Of the total 264 patients, 35 had abnormal CT scans and Miller criteria failed to detect 17 of them including 2 intoxicated (with ethanol) patients that needed neurosurgical intervention [49;50]. Another study was performed by Arienta et al. (1977) [51] on 10,000 patients in Italy. In this retrospective study, risk factors for intracranial lesions were loss of consciousness, amnesia after trauma, vomiting (repeated episodes), neurologic deficits, and signs of basal skull fracture, seizure, penetrating or perforating wounds, lack of cooperation, previous intracranial operations, coagulopathy or anticoagulant therapy, epileptic or alcoholic patients [51]. In 2003, Falimirski and colleagues [52] prospectively included 331 MTBI patients with GCS score of 14 and 15 and a history of LOC. The GCS score of 13 was excluded. The study examined patients for the presence of 10 typical constitutional signs and symptoms (CSS) for head injury including headache, somnolence, confusion, nausea/vomiting, seizure, perseveration, neurologic deficit, blurred/double vision, vertigo and hemotympanum. The results showed that loss of consciousness alone (i.e. LOC without CSS) could not predict intracranial injury. Of 195 patients without CSS, only 11 (5.6\%) had intracranial lesion and of 136 remaining patients (with CSS) $29(21.3 \%)$ had positive CT. However, it is unusual that none of the CT positive patients in both groups (with and without CSS) required neurosurgical intervention [52]. In 2007, Smits et al. [53] attempted to make a decision instrument for MTBI patients regardless of the presence or absence of a history of loss of consciousness. In a prospective study called CHIP (CT in Head Injury Patients) in four academic hospitals in the Netherlands, 3181 patients with MTBI and the GCS score of 13 and more (GCS score of 15 with at least 1 risk factor) were observed. Of 243 (7.6\%) patients with intracranial CT findings, 17 $(0.5 \%)$ patients underwent neurosurgical intervention. The major criteria were pedestrian or cyclist (vs. vehicle), ejection from vehicle, vomiting, post-traumatic amnesia for more than 4 hours, clinical signs of skull fracture, GCS score of less than15, GCS deterioration of more than 2 points, use of anticoagulants, seizure, age of more than 60 years. The minor criteria were fall from any elevation, persistent anterograde amnesia, post-traumatic amnesia for 2-4 hours, skull contusion, neurological deficit, LOC, GCS score drop of one point, age of 40-60 years. The sensitivity was $100 \%$ for neurosurgical interventions with the specificity of $23-30 \%$. For intracranial lesions, the sensitivity and specificity were $94-96 \%$ and $25-32 \%$ in the original study. The reduction rate was $23-30 \%$ [53]. The CHIP criteria were validated as a cost-effective rule. However, the annual savings were less than the CCHR rule ( $\$ 71$ million savings in CHIP vs. $120 \$$ savings in CCHR) [36]. In 2009, Saadat and colleagues [54] aimed to develop the criteria for CT scanning of MTBI patients in developing countries. Three hundred and eighteen Iranian patients with the GCS score of 13 and more were included. The major criteria were GCS score of less than 14, raccoon sign, failure to remember the impact, vomiting and age of 65 years or more. The minor criteria were wound at the scalp and GCS score of less than 15. Patients with one major criterion or 2 minor criteria had an abnormal CT scan with the sensitivity of $100 \%$ and specificity of $46 \%$ [54]. Another recent study on 642 patients in Iran showed headache, vomiting, LOC or amnesia, and alcohol intoxication as main indicators for CT scanning of MTBI patients. In the previous Iranian study, headache was not a criterion [11]. 


\begin{tabular}{|c|c|c|c|}
\hline & CCHR (medium- and high-risk) & NOC & NEXUS II \\
\hline $\begin{array}{l}\text { GCS score/Level of } \\
\text { consciousness }\end{array}$ & $<15$ at 2 hours after injury & - & $\begin{array}{l}\text { Altered level of } \\
\text { consciousness, } \\
\text { Abnormal behavior }\end{array}$ \\
\hline $\begin{array}{l}\text { Evidence of significant } \\
\text { trauma to the calvarium }\end{array}$ & $\begin{array}{l}\text { Suspected open or depressed skull } \\
\text { fracture, } \\
\text { Any sign of basal skull fracture } \\
\text { - Hemotympanum } \\
\text { - Raccoon' eyes, } \\
\text { - Cerebrospinal fluid otorrhea/ } \\
\text { rhinorrhea } \\
\text { - Battle's sign }\end{array}$ & $\begin{array}{l}\text { Physical evidence of trauma } \\
\text { above the clavicles }\end{array}$ & $\begin{array}{l}\text { Any evidence of } \\
\text { significant skull fracture, } \\
\text { Scalp hematoma }\end{array}$ \\
\hline
\end{tabular}

\begin{tabular}{llll}
\hline Vomiting & $\geq 2$ episodes & Any & Persistent \\
\hline Age & $\geq 65$ years & $>60$ years & $\geq 65$ years \\
\hline Amnesia* & $>30$ minutes, retrograde & Short-term, anterograde & - \\
\hline
\end{tabular}

Mechanism of injury* Dangerous:

- Pedestrian struck by motor

vehicle

- Occupant ejected from motor

vehicle

- Fall from height $>3$ feet or five

stairs

\begin{tabular}{llll}
\hline $\begin{array}{l}\text { Focal neurological } \\
\text { deficit }\end{array}$ & & - & Any \\
\hline Coagulopathy & - & - & Any \\
\hline Headache & - & Any & - \\
\hline Intoxication & - & Drug, Alcohol & - \\
\hline Seizure & - & Any & - \\
\hline Test sensitivity & $-100 \%[16 ; 17 ; 24 ; 30 ; 33 ; 34 ; 45]$ & $-100 \%[16 ; 17 ; 33 ; 45]$ & $-95.1 \%[45]$ \\
(neurosurgical & & $82 \%[34]$ & \\
intervention) & & & $-98.3 \%[23]$ \\
\hline Test sensitivity & $-100 \%[16 ; 33]$ & $-100 \%[16 ; 33]$ & $-97 \%[43]$ \\
(clinically important & $-98.4 \%[24]$ & $-99 \%[43]$ & $-88.7 \%[45]$ \\
intracranial injury) & -97 to 99\% [43] & $-97.7 \%$ to 99.4\% [17] & \\
& $-95 \%[34]$ & $-91.9 \%[45]$ & $-86 \%[34]$ \\
& $-83.4 \%$ to 87.2\% [17] & & $-47 \%[43]$
\end{tabular}




\begin{tabular}{llll}
\hline & CCHR (medium- and high-risk) & NOC & NEXUS II \\
\hline (clinically important & $-50.6 \%[16]$ & $-28 \%[34]$ & $-46.5 \%[45]$ \\
intracranial injury) & $-49.6 \%[24]$ & $-22.4 \%[45]$ & $-13.7 \%[23]$ \\
& -47 to $51 \%[43]$ & $-12.7 \%[16]$ & \\
& $-41.3 \%[45]$ & $-9.9 \%[33]$ & \\
& $-37.2 \%$ to $39.7 \%[17]$ & $-3.0 \%$ to $5.6 \%[17]$ & \\
& $-35 \%[33]$ & & \\
\hline
\end{tabular}

CCHR, Canadian CT head rule; NOC, New Orleans Criteria; NEXUS II, National Emergency X-Ray Utilization Study II; GCS, Glasgow Coma Scale

*Medium-risk factors for CCHR

Table 2. Comparison of three leading clinical decision rules for CT imaging in mild traumatic brain injury in adults

\section{Pediatric clinical rules}

Clinical rules for pediatric patients are less developed. It is reasonable that any of the above mentioned criteria could reduce CT imaging in pediatrics as well as adults, but further validation of pediatric head CT decision aids is needed to guarantee patients' safety [18]. In 2003, Haydel and colleagues [55] tried to determine the efficacy of the clinical rule previously developed for adults (the NOC) in 5 to 17 year-old patients. They also used the same definition of minor head injury that was used for adults. Among 175 children that were enrolled, fourteen had intracranial injury and all of them had one or more of the six following findings: headache, emesis, intoxication, seizure, short-term memory deficit and physical evidence of trauma above the clavicles. Although the sensitivity of these factors was $100 \%$, the small sample size caused a confidence interval of $74 \%$ to $100 \%$ [55]. Another study by Palchak and colleagues in 2003 [56], enrolled 2,043 children presented with traumatic brain injury (with any degree of severity) to a Level I trauma center. This observational cohort study introduced abnormal mental status, clinical signs of skull fracture, vomiting, scalp hematoma in children $\leq 2$ years of age and headache as main factors associated with traumatic brain injuries on CT scan. The rule identified all 105 patients who needed acute neurosurgical intervention and 97/98 of the patients with signs of brain injury on CT scan [56]. In 2006, NEXUS-II investigators enrolled 1,666 children including very young children ( $<3$ years) with blunt head trauma [57]. This pediatric cohort study suggested the following seven factors as the inclusion criteria for pediatrics head CT: 1 . evidence of significant skull fracture, 2. altered level of alertness, 3. neurological deficit, 4. persistent vomiting, 5. presence of scalp hematoma, 6. abnormal behavior, 7. coagulopathy. All clinically important intracranial injuries were successfully identified by the rule but two patients (in 1,666 patients) were missed. However none of them needed neurosurgical interventions [57]. Children's head injury algorithm for the prediction of important clinical events (CHALICE) is another 2006 study that was performed by Dunning et al. [58] (see table 3). A large number of children $(22,772)$ from 10 hospitals in UK with any severity of head injury were included. A sensitivity of $98 \%$ and specificity of $87 \%$ for prediction 


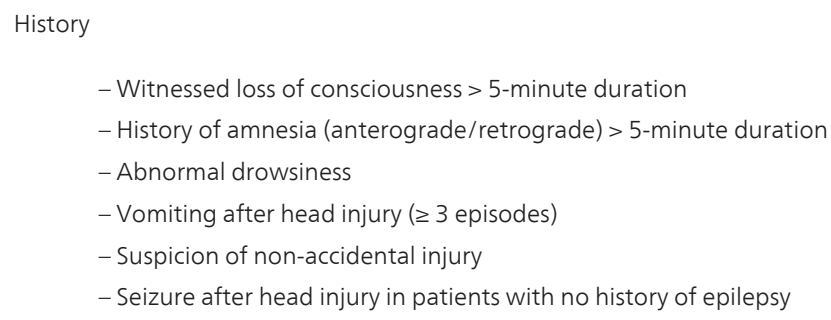

Mechanism

- High-speed road traffic accident (with speed $>40 \mathrm{~m} / \mathrm{h}$ ) either as pedestrian, cyclist or occupant

- Fall of $>3 \mathrm{~m}$ in height

- High-speed injury from a projectile or an object

Table 3. Children's head injury algorithm for the prediction of important clinical events (CHALICE). (Originally described by Dunning et al. Reproduced with permission [58])

of clinically significant head injury were documented in the study. The sensitivity was high but the rule was more comprehensive than the previous ones [58]. A retrospective survey from one hospital in Finland compared three pediatric clinical rules. NEXUS II, CHALICE and the rule presented by Palchak were compared. Each rule had 100\% sensitivity in detecting severely complicated head trauma. Severely complicated head trauma was defined as patients who required neurosurgical intervention, patients who succumbed, epidural hematoma, subdural hematoma, subarachnoid hematoma and intracerebral hematoma. NEXUS II showed the best specificity ( $21 \%$ versus $12 \%$ for Palchak and 5\% for CHALICE), although it was not high enough to detect patients who do not need neurosurgical interventions [59].

The retrospective study of Schachar and colleagues [18] on 2,101 pediatric patients (0-21 years old) has compared NOC, CCHR and NEXUS II. Sensitivities of these tests were $96.7 \%, 65.2 \%$ and $78.3 \%$, respectively. Specificities were $11.2 \%, 64.2 \%$ and $34.2 \%$, respectively. The study emphasized on the prospective ability of these tests on reduction of CT scans and concluded that further validation is needed [18].

Canadian Assessment of Tomography for Childhood Head injury (CATCH) was developed in 2010 by Osmond et al. [60]. This prospective cohort study was performed in 10 Canadian academic centers and 3,866 patients ( $0-16$ years old) with GCS score of 13 or more were 
included. High-risk factors (with 100\% sensitivity for predicting the need for neurologic intervention) include failure to reach GCS score of 15 within two hours, suspicion of open skull fracture, worsening headache and irritability. Three additional medium-risk factors (with 98.1\% sensitivity for predicting brain injury on CT scan) are large, boggy hematoma of the scalp, signs of basal skull fracture and dangerous mechanism of injury (defined as motor vehicle crash, fall from elevation $\geq 3 \mathrm{ft}$ [91 cm] or 5 stairs, fall from bicycle with no helmet) [60]. Future validation studies are needed to prove the feasibility of $\mathrm{CATCH}$.

\section{Conclusion}

In this chapter, we reviewed different clinical rules developed for patients with mild TBI. The differences among the presented guidelines are based on diversity in main outcome measures. Main outcome measure might be the following items: 1. positive brain CT scan for TBI, 2. positive clinical findings suggesting the need for neurosurgical/medical intervention, 3. a combination of the two above-mentioned findings. Brain CT scan could demonstrate small lesions related to mild TBI but it may be of a questionable practical importance. For example, the presence of a linear fracture in right frontal bone with $2 \mathrm{ml}$. of epidural hematoma below the fracture a few hours after trauma, confirms mild injury and might be valuable for legal issues, but does not predict surgical intervention requirement or even may not need anti-convulsion therapy. Therefore, if the main outcome measure was any CT findings related to the injury, we consider this case as a positive case, but if the main outcome measure was the need for neurosurgical/medical intervention, we consider the case as negative. Thus, it is important to note the fact that in many patients with traumatic brain injury on CT scan, acute neurosurgical intervention is not required and detection of intracranial injury is important when considering the need for neurosurgical intervention.

Among the above-mentioned rules, CCHR is the most validated rule with appropriate sensitivity, but the authors believe that using this rule without considering patients' history (i.e. coagulopathy, drug and alcohol intoxication) would result in missing some patients. Also progressive severe headache, focal neurological deficit and seizure should be noticed. With reference to various sensitivities and specificities documented for different clinical rules, we need more studies with this topic to reach a safe practical guideline that could be applied worldwide.

\section{Acknowledgements}

The authors would like to acknowledge Mrs. Bita Pourmand (Sina Hospital, Research Development Center) for her careful editing. 


\section{Author details}

Shayan Abdollah Zadegan ${ }^{1}$ and Vafa Rahimi-Movaghar ${ }^{1,2,3^{*}}$

*Address all correspondence to: v_rahimi@sina.tums.ac.ir; v_rahimi@yahoo.com

1 Sina Trauma and Surgery Research Center, Tehran University of Medical Sciences, Tehran, Iran

2 Department of Neurosurgery, Shariati Hospital, Tehran University of Medical Sciences, Tehran, Iran

3 Research Centre for Neural Repair, University of Tehran, Tehran, Iran

\section{References}

[1] Carroll LJ, Cassidy JD, Holm L, Kraus J, Coronado VG. Methodological issues and research recommendations for mild traumatic brain injury: the WHO Collaborating Centre Task Force on Mild Traumatic Brain Injury. J Rehabil Med 2004 Feb;(43 Suppl):113-25.

[2] McCrea M, Iverson GL, McAllister TW, Hammeke TA, Powell MR, Barr WB, et al. An integrated review of recovery after mild traumatic brain injury (MTBI): implications for clinical management. Clin Neuropsychol 2009 Nov;23(8):1368-90.

[3] Moore EL, Terryberry-Spohr L, Hope DA. Mild traumatic brain injury and anxiety sequelae: a review of the literature. Brain Inj 2006 Feb;20(2):117-32.

[4] Ruff RM, Iverson GL, Barth JT, Bush SS, Broshek DK. Recommendations for diagnosing a mild traumatic brain injury: a National Academy of Neuropsychology education paper. Arch Clin Neuropsychol 2009 Feb;24(1):3-10.

[5] Kirkwood MW, Yeates KO, Taylor HG, Randolph C, McCrea M, Anderson VA. Management of pediatric mild traumatic brain injury: a neuropsychological review from injury through recovery. Clin Neuropsychol 2008 Sep;22(5):769-800.

[6] Tavender EJ, Bosch M, Green S, O'Connor D, Pitt V, Phillips K, et al. Quality and consistency of guidelines for the management of mild traumatic brain injury in the emergency department. Acad Emerg Med 2011 Aug;18(8):880-9.

[7] Tellier A, la Malva LC, Cwinn A, Grahovac S, Morrish W, Brennan-Barnes M. Mild head injury: a misnomer. Brain Inj 1999 Jul;13(7):463-75.

[8] Kushner D. Mild traumatic brain injury: toward understanding manifestations and treatment. Arch Intern Med 1998 Aug 10;158(15):1617-24. 
[9] Washington CW, Grubb RL, Jr. Are routine repeat imaging and intensive care unit admission necessary in mild traumatic brain injury? J Neurosurg 2012 Mar;116(3): 549-57.

[10] Zare MA, Ahmadi K, Zadegan SA, Farsi D, Rahimi-Movaghar V. Effects of brain contusion on mild traumatic brain-injured patients. Int J Neurosci 2013 Jan;123(1):65-9.

[11] Sharif-Alhoseini M, Khodadadi H, Chardoli M, Rahimi-Movaghar V. Indications for brain computed tomography scan after minor head injury. J Emerg Trauma Shock 2011 Oct;4(4):472-6.

[12] Ruff RM. Mild traumatic brain injury and neural recovery: rethinking the debate. NeuroRehabilitation 2011;28(3):167-80.

[13] Jagoda AS, Bazarian JJ, Bruns JJ, Jr., Cantrill SV, Gean AD, Howard PK, et al. Clinical policy: neuroimaging and decisionmaking in adult mild traumatic brain injury in the acute setting. J Emerg Nurs 2009 Apr;35(2):e5-40.

[14] Mittenberg W, Canyock EM, Condit D, Patton C. Treatment of post-concussion syndrome following mild head injury. J Clin Exp Neuropsychol 2001 Dec;23(6):829-36.

[15] Haydel MJ, Preston CA, Mills TJ, Luber S, Blaudeau E, DeBlieux PM. Indications for computed tomography in patients with minor head injury. N Engl J Med $2000 \mathrm{Jul}$ $13 ; 343(2): 100-5$.

[16] Stiell IG, Clement CM, Rowe BH, Schull MJ, Brison R, Cass D, et al. Comparison of the Canadian CT Head Rule and the New Orleans Criteria in patients with minor head injury. JAMA 2005 Sep 28;294(12):1511-8.

[17] Smits M, Dippel DW, de Haan GG, Dekker HM, Vos PE, Kool DR, et al. External validation of the Canadian CT Head Rule and the New Orleans Criteria for CT scanning in patients with minor head injury. JAMA 2005 Sep 28;294(12):1519-25.

[18] Schachar JL, Zampolin RL, Miller TS, Farinhas JM, Freeman K, Taragin BH. External validation of the New Orleans Criteria (NOC), the Canadian CT Head Rule (CCHR) and the National Emergency X-Radiography Utilization Study II (NEXUS II) for CT scanning in pediatric patients with minor head injury in a non-trauma center. Pediatr Radiol 2011 Aug;41(8):971-9.

[19] Mack LR, Chan SB, Silva JC, Hogan TM. The use of head computed tomography in elderly patients sustaining minor head trauma. J Emerg Med 2003 Feb;24(2):157-62.

[20] Ibanez J, Arikan F, Pedraza S, Sanchez E, Poca MA, Rodriguez D, et al. Reliability of clinical guidelines in the detection of patients at risk following mild head injury: results of a prospective study. J Neurosurg 2004 May;100(5):825-34.

[21] Fabbri A, Servadei F, Marchesini G, Dente M, Iervese T, Spada M, et al. Clinical performance of NICE recommendations versus NCWFNS proposal in patients with mild head injury. J Neurotrauma 2005 Dec;22(12):1419-27. 
[22] Iverson GL, Lovell MR, Smith S, Franzen MD. Prevalence of abnormal CT-scans following mild head injury. Brain Inj 2000 Dec;14(12):1057-61.

[23] Mower WR, Hoffman JR, Herbert M, Wolfson AB, Pollack CV, Jr., Zucker MI. Developing a decision instrument to guide computed tomographic imaging of blunt head injury patients. J Trauma 2005 Oct;59(4):954-9.

[24] Stiell IG, Wells GA, Vandemheen K, Clement C, Lesiuk H, Laupacis A, et al. The Canadian CT Head Rule for patients with minor head injury. Lancet 2001 May 5;357(9266):1391-6.

[25] Boyle A, Santarius L, Maimaris C. Evaluation of the impact of the Canadian CT head rule on British practice. Emerg Med J 2004 Jul;21(4):426-8.

[26] Rosengren D, Rothwell S, Brown AF, Chu K. The application of North American CT scan criteria to an Australian population with minor head injury. Emerg Med Australas 2004 Jun;16(3):195-200.

[27] Sultan HY, Boyle A, Pereira M, Antoun N, Maimaris C. Application of the Canadian CT head rules in managing minor head injuries in a UK emergency department: implications for the implementation of the NICE guidelines. Emerg Med J 2004 Jul; 21(4):420-5.

[28] van Essen TA, Heeringa JJ, Muizelaar JP. (In)appropriate neurosurgical consultation. Clin Neurol Neurosurg 2010 Nov;112(9):775-80.

[29] Syed AT, Lone NA, Wani MA, Bhat AS. Clinical management of patients with minor head injuries. Int J Health Sci (Qassim ) 2007 Jan;1(1):131-40.

[30] Clement CM, Stiell IG, Schull MJ, Rowe BH, Brison R, Lee JS, et al. Clinical features of head injury patients presenting with a Glasgow Coma Scale score of 15 and who require neurosurgical intervention. Ann Emerg Med 2006 Sep;48(3):245-51.

[31] Fong C, Chong W, Villaneuva E, Segal AY. Implementation of a guideline for computed tomography head imaging in head injury: a prospective study. Emerg Med Australas 2008 Oct;20(5):410-9.

[32] Perry JJ, Stiell IG. Impact of clinical decision rules on clinical care of traumatic injuries to the foot and ankle, knee, cervical spine, and head. Injury 2006 Dec;37(12): 1157-65.

[33] Papa L, Stiell IG, Clement CM, Pawlowicz A, Wolfram A, Braga C, et al. Performance of the Canadian CT Head Rule and the New Orleans Criteria for predicting any traumatic intracranial injury on computed tomography in a United States Level I trauma center. Acad Emerg Med 2012 Jan;19(1):2-10.

[34] Bouida W, Marghli S, Souissi S, Ksibi H, Methammem M, Haguiga H, et al. Prediction Value of the Canadian CT Head Rule and the New Orleans Criteria for Positive 
Head CT Scan and Acute Neurosurgical Procedures in Minor Head Trauma: A Multicenter External Validation Study. Ann Emerg Med 2013 May;61(5):521-7.

[35] Holmes MW, Goodacre S, Stevenson MD, Pandor A, Pickering A. The cost-effectiveness of diagnostic management strategies for adults with minor head injury. Injury 2012 Sep;43(9):1423-31.

[36] Smits M, Dippel DW, Nederkoorn PJ, Dekker HM, Vos PE, Kool DR, et al. Minor head injury: CT-based strategies for management--a cost-effectiveness analysis. Radiology 2010 Feb;254(2):532-40.

[37] Stiell IG, Clement CM, Grimshaw JM, Brison RJ, Rowe BH, Lee JS, et al. A prospective cluster-randomized trial to implement the Canadian CT Head Rule in emergency departments. CMAJ 2010 Oct 5;182(14):1527-32.

[38] Stiell IG, Bennett C. Implementation of clinical decision rules in the emergency department. Acad Emerg Med 2007 Nov;14(11):955-9.

[39] Curran JA, Brehaut J, Patey AM, Osmond M, Stiell I, Grimshaw JM. Understanding the Canadian adult CT head rule trial: use of the theoretical domains framework for process evaluation. Implement Sci 2013 Feb 21;8:25. doi: 10.1186/1748-5908-8-25.:25-8.

[40] Eagles D, Stiell IG, Clement CM, Brehaut J, Taljaard M, Kelly AM, et al. International survey of emergency physicians' awareness and use of the Canadian Cervical-Spine Rule and the Canadian Computed Tomography Head Rule. Acad Emerg Med 2008 Dec;15(12):1256-61.

[41] Bruns JJ, Jr., Jagoda AS. Mild traumatic brain injury. Mt Sinai J Med 2009 Apr;76(2): 129-37.

[42] Harnan SE, Pickering A, Pandor A, Goodacre SW. Clinical decision rules for adults with minor head injury: a systematic review. J Trauma 2011 Jul;71(1):245-51.

[43] Stein SC, Fabbri A, Servadei F, Glick HA. A critical comparison of clinical decision instruments for computed tomographic scanning in mild closed traumatic brain injury in adolescents and adults. Ann Emerg Med 2009 Feb;53(2):180-8.

[44] Mower WR, Hoffman JR, Herbert M, Wolfson AB, Pollack CV, Jr., Zucker MI. Developing a clinical decision instrument to rule out intracranial injuries in patients with minor head trauma: methodology of the NEXUS II investigation. Ann Emerg Med 2002 Nov;40(5):505-14.

[45] Ro YS, Shin SD, Holmes JF, Song KJ, Park JO, Cho JS, et al. Comparison of clinical performance of cranial computed tomography rules in patients with minor head injury: a multicenter prospective study. Acad Emerg Med 2011 Jun;18(6):597-604.

[46] Reinus WR, Wippold FJ, Erickson KK. Practical selection criteria for noncontrast cranial computed tomography in patients with head trauma. Ann Emerg Med 1993 Jul; 22(7):1148-55. 
[47] Duus BR, Lind B, Christensen H, Nielsen OA. The role of neuroimaging in the initial management of patients with minor head injury. Ann Emerg Med 1994 Jun;23(6): 1279-83.

[48] Borczuk P. Predictors of intracranial injury in patients with mild head trauma. Ann Emerg Med 1995 Jun;25(6):731-6.

[49] Miller EC, Holmes JF, Derlet RW. Utilizing clinical factors to reduce head CT scan ordering for minor head trauma patients. J Emerg Med 1997 Jul;15(4):453-7.

[50] Holmes JF, Baier ME, Derlet RW. Failure of the Miller criteria to predict significant intracranial injury in patients with a Glasgow Coma Scale score of 14 after minor head trauma. Acad Emerg Med 1997 Aug;4(8):788-92.

[51] Arienta C, Caroli M, Balbi S. Management of head-injured patients in the emergency department: a practical protocol. Surg Neurol 1997 Sep;48(3):213-9.

[52] Falimirski ME, Gonzalez R, Rodriguez A, Wilberger J. The need for head computed tomography in patients sustaining loss of consciousness after mild head injury. J Trauma 2003 Jul;55(1):1-6.

[53] Smits M, Dippel DW, Steyerberg EW, de Haan GG, Dekker HM, Vos PE, et al. Predicting intracranial traumatic findings on computed tomography in patients with minor head injury: the CHIP prediction rule. Ann Intern Med 2007 Mar;\%20;146(6): 397-405.

[54] Saadat S, Ghodsi SM, Naieni KH, Firouznia K, Hosseini M, Kadkhodaie HR, et al. Prediction of intracranial computed tomography findings in patients with minor head injury by using logistic regression. J Neurosurg 2009 Oct;111(4):688-94.

[55] Haydel MJ, Shembekar AD. Prediction of intracranial injury in children aged five years and older with loss of consciousness after minor head injury due to nontrivial mechanisms. Ann Emerg Med 2003 Oct;42(4):507-14.

[56] Palchak MJ, Holmes JF, Vance CW, Gelber RE, Schauer BA, Harrison MJ, et al. A decision rule for identifying children at low risk for brain injuries after blunt head trauma. Ann Emerg Med 2003 Oct;42(4):492-506.

[57] Oman JA, Cooper RJ, Holmes JF, Viccellio P, Nyce A, Ross SE, et al. Performance of a decision rule to predict need for computed tomography among children with blunt head trauma. Pediatrics 2006 Feb;117(2):e238-e246.

[58] Dunning J, Daly JP, Lomas JP, Lecky F, Batchelor J, kway-Jones K. Derivation of the children's head injury algorithm for the prediction of important clinical events decision rule for head injury in children. Arch Dis Child 2006 Nov;91(11):885-91.

[59] Klemetti S, Uhari M, Pokka T, Rantala H. Evaluation of decision rules for identifying serious consequences of traumatic head injuries in pediatric patients. Pediatr Emerg Care 2009 Dec;25(12):811-5. 
[60] Osmond MH, Klassen TP, Wells GA, Correll R, Jarvis A, Joubert G, et al. CATCH: a clinical decision rule for the use of computed tomography in children with minor head injury. CMAJ 2010 Mar 9;182(4):341-8. 